ARTICLE

https://doi.org/10.1038/s41467-018-07753-2

\title{
Intron-containing RNA from the HIV-1 provirus activates type I interferon and inflammatory cytokines
}

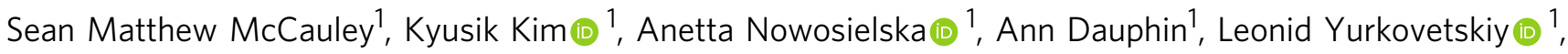 \\ William Edward Diehl (1) ${ }^{1}$ \& Jeremy Luban (10) ${ }^{1,2}$
}

HIV-1-infected people who take drugs that suppress viremia to undetectable levels are protected from developing AIDS. Nonetheless, HIV-1 establishes proviruses in long-lived CD4 ${ }^{+}$ memory $T$ cells, and perhaps other cell types, that preclude elimination of the virus even after years of continuous antiviral therapy. Here we show that the HIV-1 provirus activates innate immune signaling in isolated dendritic cells, macrophages, and $\mathrm{CD}^{+}{ }^{+} \mathrm{T}$ cells. Immune activation requires transcription from the HIV-1 provirus and expression of CRM1-dependent, Revdependent, RRE-containing, unspliced HIV-1 RNA. If rev is provided in trans, all HIV-1 coding sequences are dispensable for activation except those cis-acting sequences required for replication or splicing. Our results indicate that the complex, post-transcriptional regulation intrinsic to HIV-1 RNA is detected by the innate immune system as a danger signal, and that drugs which disrupt HIV-1 transcription or HIV-1 RNA metabolism would add qualitative benefit to current antiviral drug regimens.

\footnotetext{
${ }^{1}$ Program in Molecular Medicine, University of Massachusetts Medical School, Worcester, MA 01605, USA. ${ }^{2}$ Department of Biochemistry and Molecular Pharmacology, University of Massachusetts Medical School, Worcester, MA 01605, USA. Correspondence and requests for materials should be addressed to J.L. (email: jeremy.luban@umassmed.edu)
} 
T here are currently over 40 antiretroviral drug formulations that block essential steps in the HIV-1 replication cycle, including reverse transcription, integration, and proteolytic processing of the viral polyproteins ${ }^{1,2}$. Although these treatments are effective at reducing viremia, HIV-1 establishes proviruses in long-lived $\mathrm{CD}^{+}$memory T cells, and perhaps other cell types ${ }^{3-5}$, that preclude elimination of the virus even after years of continuous antiviral therapy ${ }^{6}$. As a result, HIV-1-infected individuals must maintain a strict regimen of antiviral drugs or risk viral rebound and development of HIV-1 drug resistance ${ }^{6-9}$. Furthermore, despite the drastic reduction in viral replication, these individuals sometimes exhibit chronic inflammation associated with heightened risk of cardiovascular pathology $y^{10-12}$. Though the majority of proviruses that persist during antiviral therapy are defective for the production of infectious virions ${ }^{13}$, many proviruses are expressed, raising the possibility that the HIV-1 provirus, or its transcripts, contribute to ongoing inflammation ${ }^{14}$.

HIV-1 exploits a number of host and viral factors to avoid detection by the innate immune system ${ }^{15}$. Innate immune detection of HIV-1 has been reported to occur via a variety of mechanisms. Early after encounter with a target cell, HIV-1 CA interaction with host TRIM5 ${ }^{16,17}$, or, independently, HIV-1 gp $41^{18}$, activates transforming growth factor beta-activated kinase 1 (TAK1)-dependent signaling pathways. Plasmacytoid dendritic cells (pDCs) readily detect HIV-1 RNA via TLR7 and produce copious levels of type I interferon ${ }^{19-21}$. HIV-1 genomic RNA has also been reported to activate RIG-I signaling 22,23 , and HIV-1 cDNA can be detected by the cytoplasmic DNA sensor cGAS $^{24-26}$. Sensing of HIV-1 infection has also been suggested to occur following integration, with critical determinants including HIV-1 capsid ${ }^{27}$, post-integration cGAS signaling ${ }^{25}$, and abortive HIV-1 RNA transcripts ${ }^{28}$.

The determinants for innate immune detection of HIV-1 were investigated in detail here. To start, dendritic cells were challenged with a large panel of single-cycle HIV-1 vectors. The results show that high-level activation of innate immune gene expression in transduced cells requires integration, as well as transcription from the provirus. Furthermore, DC maturation is dependent upon Rev-CRM1-mediated export of intron-bearing, HIV-1 genomic RNA. Transduced macrophages and $\mathrm{CD} 4{ }^{+}$ $\mathrm{T}$ cells behave like transduced DCs. Primary HIV-1 clones in the context of complete, replication-competent HIV-1 also give similar results. The observations reported here demonstrate that the innate immune system detects HIV-1's unique modes of RNA processing and suggest that inhibitors of HIV-1 transcription or HIV-1 RNA metabolism would be beneficial additions to current retroviral therapy.

\section{Results}

Dendritic cells mature in response to challenge with HIV-1. To determine if HIV-1 proviruses activate innate immune signaling, human blood cells were transduced with single-cycle vectors, either a full-length, single-cycle HIV-1 clone with a frameshift in $e n v$ and eGFP in place of nef (HIV-1-GFP) ${ }^{29}$, or a minimal 3-part lentivector encoding GFP (Fig. 1a, and Supplementary Table 1) ${ }^{16}$. Monocyte derived dendritic cells (DCs) were challenged initially since HIV-1 transduction of these specialized antigen-presenting cells activates innate immune signaling 23,24,27,30,31. To increase the efficiency of provirus establishment, vectors were pseudotyped with the vesicular stomatitis virus glycoprotein (VSV G) and delivered concurrently with virus-like particles (VLPs) bearing $\operatorname{SIV}_{\mathrm{MAC}} 251 \mathrm{Vpx}$ (Fig. 1b) ${ }^{16,32}$. Transduction efficiency, as determined by flow cytometry for GFP-positive cells, was 30-60\% (Fig. 1b and Supplementary Fig 1a), depending on the blood donor.
DCs matured in response to HIV-1-GFP transduction, as indicated by increased mean fluorescence intensity of costimulatory or activation molecules, including HLA-DR, CD80, CD86, CD40, CD83, CCR7, CD141, ISG15, MX1, and IFIT1 ${ }^{33}$ (Fig. 1b, c and Supplementary Fig. 1a, b). Identical results were obtained with full-length, single-cycle vectors generated from primary, transmitted/founder clones that were derived by single genome sequencing, HIV- $1_{\mathrm{AD} 17^{34}}$, HIV- $1_{\mathrm{Z} 331 \mathrm{M}-\mathrm{TF}}{ }^{35}$, and HIV$1_{\mathrm{ZM} 249 \mathrm{M}^{36}}$, the first virus being clade $\mathrm{B}$, the other two clade $\mathrm{C}$ (Fig. 1d and Supplementary Fig. 1c). A single cycle HIV-2 vector induced maturation, indicating that this innate response was not unique to HIV-1 (Fig. 1e and Supplementary Fig. 1d).

Maturation was evident among both GFP positive and negative cells (Fig. 1b), the latter likely resulting from activation in trans by type 1 IFN as others have shown ${ }^{27,31}$. To test this idea, naive DCs were incubated with filtered supernatant from autologous DCs that had been transduced previously with HIV-1-GFP. Supernatant from DCs transduced with HIV-1-GFP, but not with minimal lentivector, upregulated CD86 and ISG15 on the naive DCs (Fig. If and Supplementary Fig 1e). Maturation activity was evident at a 1:1000 dilution of supernatant to which nevirapine had been added to preclude carry-over of transductioncompetent HIV-1-GFP.

DCs matured when HIV-1-GFP transduction efficiency was augmented with nucleosides ${ }^{37}$, rather than with SIV VLPs, indicating that $\mathrm{Vpx}$ was not required for maturation (Fig. $1 \mathrm{~g}$ and Supplementary Fig. 1f). DCs were then challenged with replication-competent HIV-1 bearing CCR5-tropic Env, either T cell-tropic or macrophage-tropic ${ }^{38}$, with or without Vpx-VLPs (Fig. $1 \mathrm{~h}$ and Supplementary Fig. 1g). The percent of cells transduced by vector bearing either Env increased with Vpx, though DC maturation was observed under all conditions, even among the very few DCs transduced by T cell-tropic env (see inset of Fig. 1h). These results indicate that neither VSV G, nor Vpx, nor high-titer infection, was required for DC maturation.

In response to transduction with HIV-1-GFP, steady-state CXCL10, IFNB1, and IL15 mRNAs reached maximum levels at 48 $\mathrm{h}$, increasing 31,000-, 92-, and 140-fold relative to mock-treated cells, respectively (Fig. 1i, j). Correspondingly, IFNa2, CCL7, IL-6, CXCL10, and TNFa proteins accumulated in the supernatant (Fig. 1k). In contrast to the results with HIV-1-GFP, there were no signs of maturation after transduction with the 3-part minimal lentivector (Fig. 1b, j, k and Supplementary Fig. 1a, b, c, d).

DC maturation requires reverse transcription and integration. To determine if early stages in the HIV-1 replication cycle were necessary for maturation, reverse transcription was inhibited by nevirapine (NVP) or the HIV-1 RT mutant D185K/D186L, and integration was inhibited with raltegravir (RALT) or the HIV-1 IN mutant D116A (Supplementary Tables 1 and 2), as previously described ${ }^{39}$. Each of these four conditions abrogated maturation, as indicated by cell surface CD86 (Fig. 2a, and Supplementary Fig. 2a) and steady-state CXCL10 mRNA (Fig. 2b). When integration was inhibited, CXCL10 mRNA increased in response to challenge with HIV-1-GFP, but levels were nearly 1,000 times lower than when integration was not blocked (Fig. 2b). As an additional control, DCs challenged with the well-characterized, non-infectious, protease active-site mutant D25 $\mathrm{A}^{40}$ expressed 10,000 times less CXCL10 mRNA than did cells transduced with HIV-1-GFP (Fig. 2b).

HIV-1 virion RNA and newly synthesized viral cDNA are reported to be detected by RIG-I and by cGAS, respectively ${ }^{23,24}$. Signal transduction downstream of both sensors requires TBK1 and IRF3. The TBK1 inhibitor BX795 (Supplementary Table 2) blocked DC maturation in response to cGAMP but had no effect on maturation after HIV-1-GFP transduction (Fig. 2c and 
a

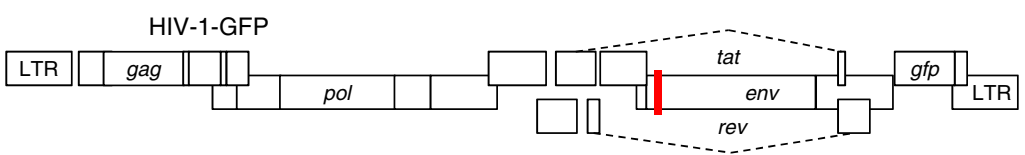

\begin{tabular}{|c|c|c|c|c|c|c|c|}
\hline \multicolumn{5}{|c|}{ Lentivector } & \multicolumn{3}{|c|}{$\rightarrow$} \\
\hline CMV Pro & $\psi$ & RRE & $\begin{array}{lc}\text { CPPT } \\
\end{array}$ & SFFV & $g f p$ & WPRE & $\Delta \mathrm{U} 3$ \\
\hline
\end{tabular}

b Untreated DCs

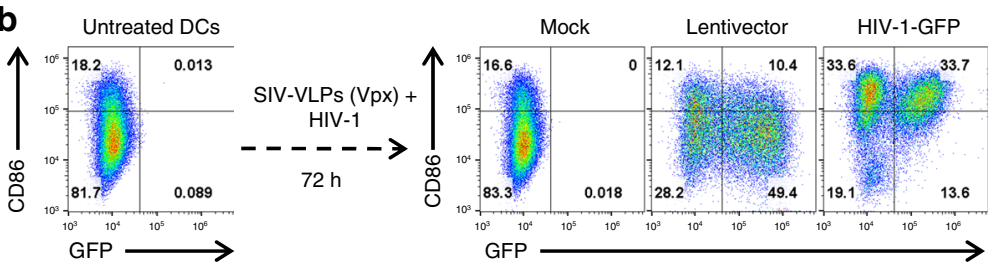

C
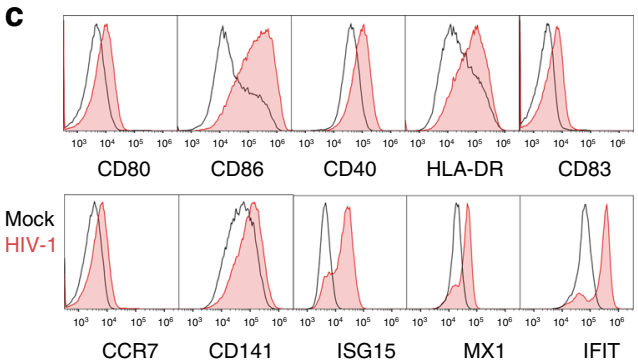

d

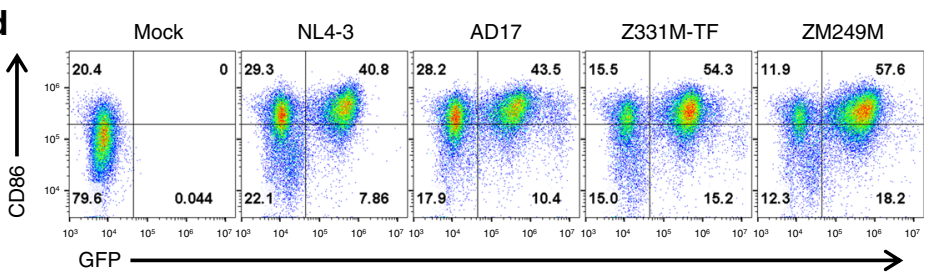

e

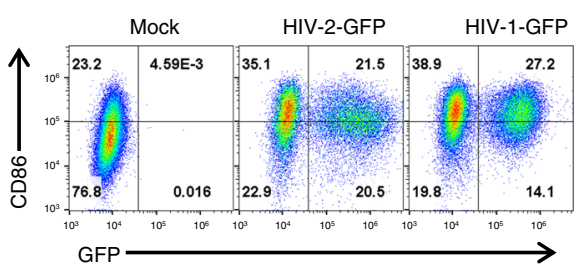

f

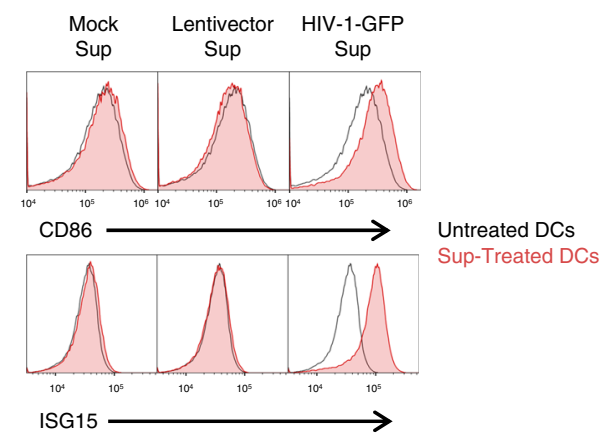

g

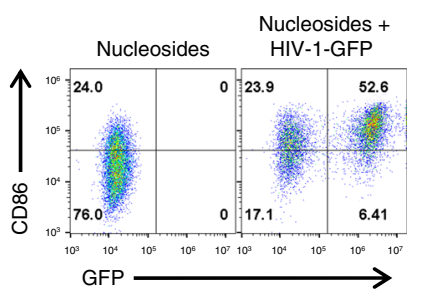

h
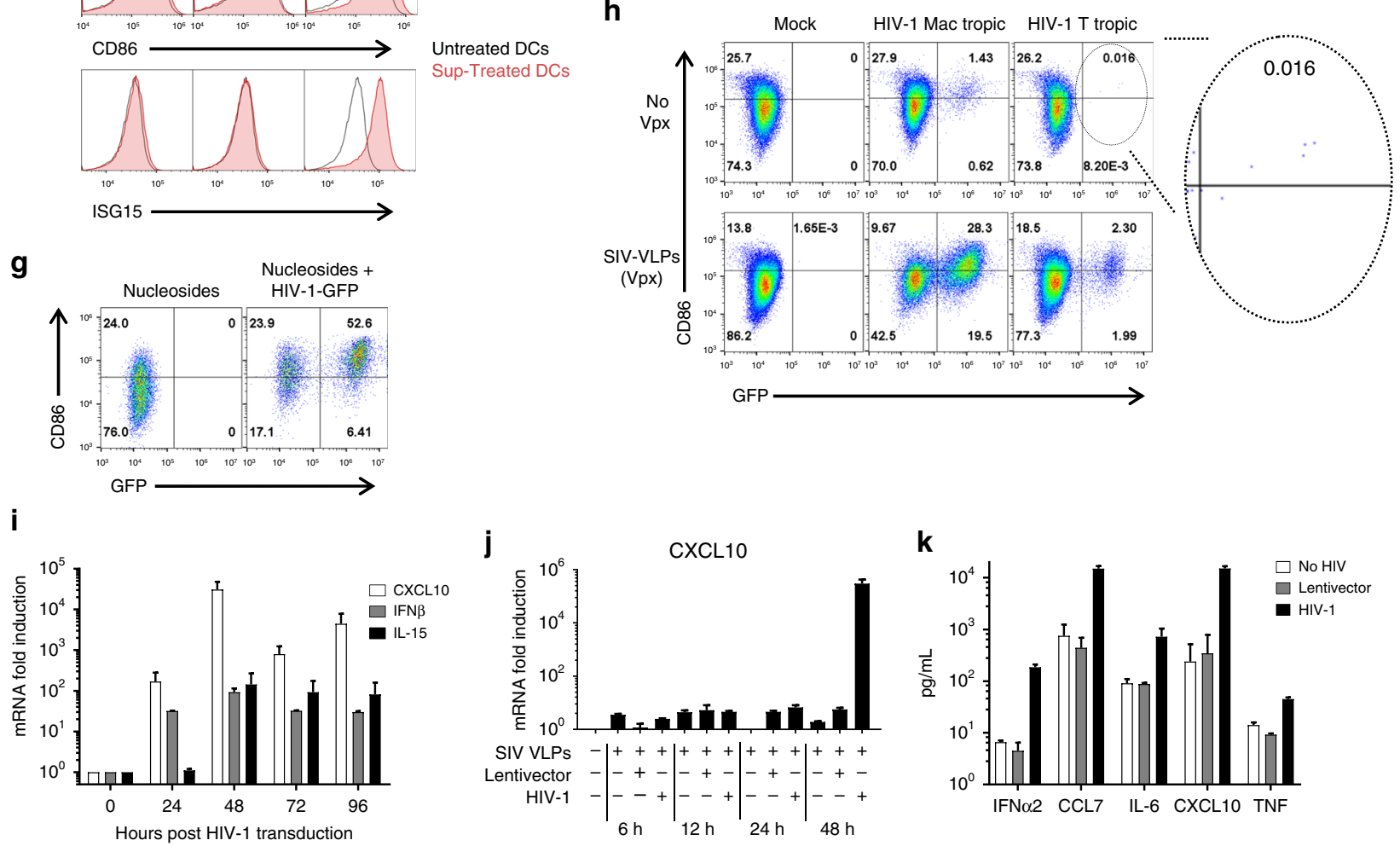

Supplementary Fig. 2b). Moreover, IRF3 knockdown (Supplementary Table 1) ${ }^{16}$ suppressed activation of CD86 or ISG15 in response to CGAMP, but not in response to HIV-1 transduction (Fig. 2c and Supplementary Fig. 2b). Similarly, no effect on HIV1 -induced DC maturation was observed with knockdown of IRF1, $5,7$, or 9 , or of STAT1 or 2, Supplementary Table 1$)^{16}$, or of pharmacologic inhibition of CypA, PKR, c-Raf, IkBa, NF-kB, MEK1 + 2, p38, JNK, Caspase 1, pan-Caspases, ASK1, eIF2a,
TBK1, IKKe, TAK1, or NLRP3 (Supplementary Table 2). Under the conditions used here, then, DC maturation required reverse transcription and integration but was independent of most well characterized innate immune signaling pathways.

Provirus transcription is required for innate activation. Completion of the HIV-1 integration reaction requires cellular DNA 
Fig. 1 HIV-1 transduction matures DCs. a Schematic of HIV-1-GFP, with frameshift in env (red line) and gfp in place of nef ${ }^{29}$, and of the minimal lentivector, with self-inactivating $\Delta \mathrm{U} 3 \mathrm{LTR}^{42}$ and $g f p$ driven by the SFFV promoter ${ }^{16}$. Unless indicated otherwise, vectors were pseudotyped with VSV G and cells were

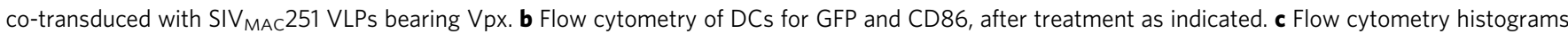
for the indicated markers $72 \mathrm{~h}$ after DC transduction with HIV-1 (red) or mock (black). d Flow cytometry of DCs for GFP and CD86 after transduction with

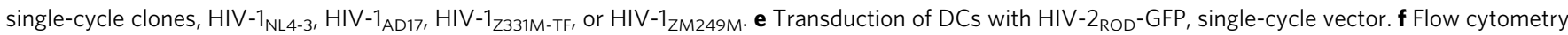
for CD86 and ISG15 of DCs treated for $24 \mathrm{~h}$ in the presence of nevirapine with a 1:1000 dilution of supernatant from autologous DCs transduced with the indicated vectors. $\mathbf{g}$ DC transduction with HIV-1-GFP in the absence of $\mathrm{Vpx}$ and the presence of $2 \mathrm{mM}$ nucleosides. $\mathbf{h} 12$-day spreading infection on DCs, with macrophage-tropic or T cell-tropic, replication-competent HIV-1, with or without SIV VLPs. i qRT-PCR quantitation of CXCL10 (black), IFNB1 (gray), or IL15 (white) mRNAs from DCs transduced with HIV-1-GFP. $\mathbf{j}$ qRT-PCR quantitation of CXCL10 mRNA in DCs transduced with either HIV-1-GFP or minimal lentivector, assessed at the indicated times post-transduction. $\mathbf{k}$ Cytokines in DC supernatant as assessed by luminex, $72 \mathrm{~h}$ after transduction with HIV-1GFP (black) or minimal lentivector (gray). Shown are blood donor data representative of $n=12(\mathbf{b}), n=4$ (c-k), or $n=8$ (h). To determine significance, the MFI of all live cells for each sample was calculated as fold-change versus control. The exception being $\mathbf{h}$ where the MFI of only GFP+ cells was compared. When data from each donor replicate within a experiment was combined, the difference in MFI for all experimental vs control conditions was significant in all cases, $p<0.01$; one-way ANOVA, Dunnett's post-test. qRT-PCR and Luminex data were mean \pm SD, $p<0.0001$; two-way ANOVA, Dunnett's post-test

repair enzymes ${ }^{41}$. That DCs did not mature in response to transduction with minimal lentivectors (Fig. $1 \mathrm{~b}, \mathrm{j}, \mathrm{k}$ ) indicates that activation of the DNA repair process is not sufficient, and that transcription from the HIV-1-GFP provirus must be necessary for maturation. Indeed, gag expression from an integrated vector has been reported to be necessary for DC maturation ${ }^{27}$. To determine if any individual HIV-1 proteins were sufficient to mature DCs, a minimal lentivector was used to express codon optimized versions of each of the open reading frames possessed by HIV-1-GFP (Fig. 2d, Supplementary Fig. 2b, and Supplementary Table 1). Among these vectors was a gag-expression vector that produced as much p24 protein as did HIV-1-GFP (Fig. 2d). None of these vectors matured DCs (Fig. 2d and Supplementary Fig. 2d).

HIV-1-GFP was then mutated to determine if any protein coding sequences were necessary for DC maturation. For these and any subsequent experiments in which an essential viral component was disrupted within HIV-1-GFP, the factor in question was provided in trans, either during assembly in transfected HEK293 cells, or within transduced DCs, as appropriate (see Methods). Mutations that disrupted both gag and pol, either a double frameshift in gag, or a mutant in which the first 14 ATGs in gag were mutated, abolished synthesis of CA (p24) yet retained full maturation activity (Fig. 2e, Supplementary Fig. 2e, and Supplementary Table 1). Deletion mutations encompassing gag/pol, vif/vpr, vpu/env, or nef/U3-LTR, each designed so as to leave cis-acting RNA elements intact, all matured DCs (Fig. 2f, Supplementary Fig. 2e, and Supplementary Table 1). These results indicate that these HIV-1-GFP RNA sequences, as well as the proteins that they encode, were not required for DC maturation.

Tat and Rev coding sequences were individually disrupted by combining start codon point mutations with nonsense codons that were silent with respect to overlapping reading frames (Supplementary Table 1). Neither $\Delta$ tat nor $\Delta$ rev matured DCs upon transduction (Fig. 2g and Supplementary Fig. 2f). However, DCs matured upon co-transduction of $\Delta$ tat and $\Delta r e v$, or when minimal lentivectors expressing codon-optimized Tat and Rev were co-transduced in trans (Fig. $2 \mathrm{~g}$ and Supplementary Fig. 2g). These results indicate that the maturation defect with the individual vectors was due to disruption of Tat and Rev function, and not due to a cis-acting defect of the mutant RNA.

The minimal 3-part lentivector expressed GFP from a heterologous promoter and had a deletion mutation encompassing the essential, cis-acting TATA box and enhancer elements ${ }^{42}$, as well as in the trans-acting tat and rev, that inactivated the promoter in the proviral 5' LTR (Fig. 1a). To test the importance of LTR-driven transcription for DC maturation by the HIV-1 provirus, the HIV-1 LTR was restored in the minimal vector (Fig. 2h, Supplementary Fig. 2g, and Supplementary Table 1); in addition, GFP was inserted in place of $g a g$ as a marker for LTR expression, and the heterologous promoter was used to drive tat, rev, or both genes separated by P2A coding sequence (Fig. 2h). None of the LTR-driven, minimal vectors matured DCs (Fig. $2 \mathrm{~h}$ and Supplementary Fig. $2 \mathrm{~g}$ ).

To determine if tat was necessary for DC maturation, tat and TAR were mutated in HIV-1-GFP and the LTR promoter was modified to be tetracycline-inducible, as previously described ${ }^{43}$ (Tet-HIV-1 in Fig. $3 \mathrm{a}$ and Supplementary Table 1). The doxycycline-dependent reverse transactivator $(r t T A)$ was delivered in trans by lentivector. In the presence of doxycycline (Supplementary Table 2), Tet-HIV-1 and rtTA matured DCs when given in combination, but neither vector matured DCs when given in isolation (Fig. 3a and Supplementary Fig. 3a). Additionally, the magnitude of cell surface CD86 was dependent on the doxycycline concentration, indicating that maturation was dependent on the level of HIV-1 transcription (Fig. $3 \mathrm{~b}$ and Supplementary Fig. 3b). These results demonstrated that tat was not required for maturation, so long as the provirus was expressed.

Rev-CRM1-mediated RNA export is necessary for activation. To ascertain whether rev was necessary for DC maturation, the RTE from a murine intracisternal A-particle retroelement (IAP), and the CTE from SRV-1, were inserted in place of nef (HIVRTE/CTE in Fig. 3c, Supplementary Fig. 3c, and Supplementary Table 1$)^{44}$. Each of these elements utilizes the NXF1 nuclear RNA export pathway, thereby bypassing the need for CRM1 and $r e v^{45}$. p24 levels with this construct were similar to those of HIV-1GFP, indicating that unspliced RNA was exported from the nucleus at least as well as with Rev (Fig. 3c). Nonetheless, the HIV-RTE/CTE vector did not mature DCs (Fig. $3 \mathrm{c}$ and Supplementary Fig. 3c), indicating that maturation was dependent upon rev and CRM1-mediated RNA export. Consistent with this conclusion, the CRM1 inhibitor leptomycin B (Supplementary Table 2) abrogated DC maturation by HIV-1-GFP (Fig. 3d and Supplementary Fig. 3d). In contrast, leptomycin B had no effect on DC maturation in response to Sendai virus infection (Fig. 3e and Supplementary Fig. 3d). ISG15 was used to monitor maturation in these experiments since, as previously reported for DCs, leptomycin B altered background levels of CD86 ${ }^{46}$.

HIV-1 activates macrophages and $\mathrm{CD}^{+} \mathrm{T}$ cells. To determine if innate immune detection of HIV-1 was unique to DCs, monocytederived macrophages and $\mathrm{CD}^{+}{ }^{+} \mathrm{T}$ cells were examined. In response to transduction with HIV-1-GFP, macrophages upregulated CD86, ISG15, and HLA-DR, and CD4 ${ }^{+} \mathrm{T}$ cells upregulated MX1, IFIT1, and HLA-DR (Fig. 4a and Supplementary Fig. 4a-f). DCs, macrophages, and $\mathrm{CD}^{+} \mathrm{T}$ cells were then transduced side-by-side with mutant constructs to determine if the 


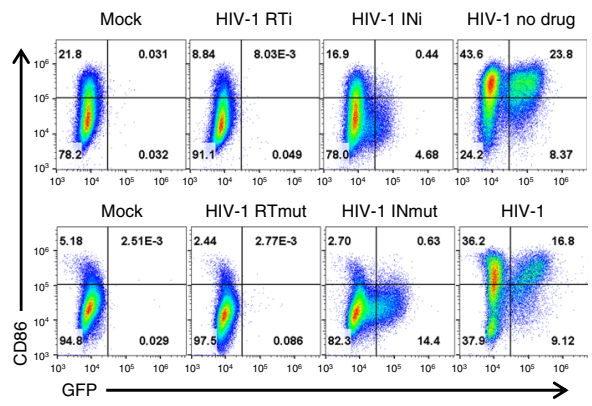

b
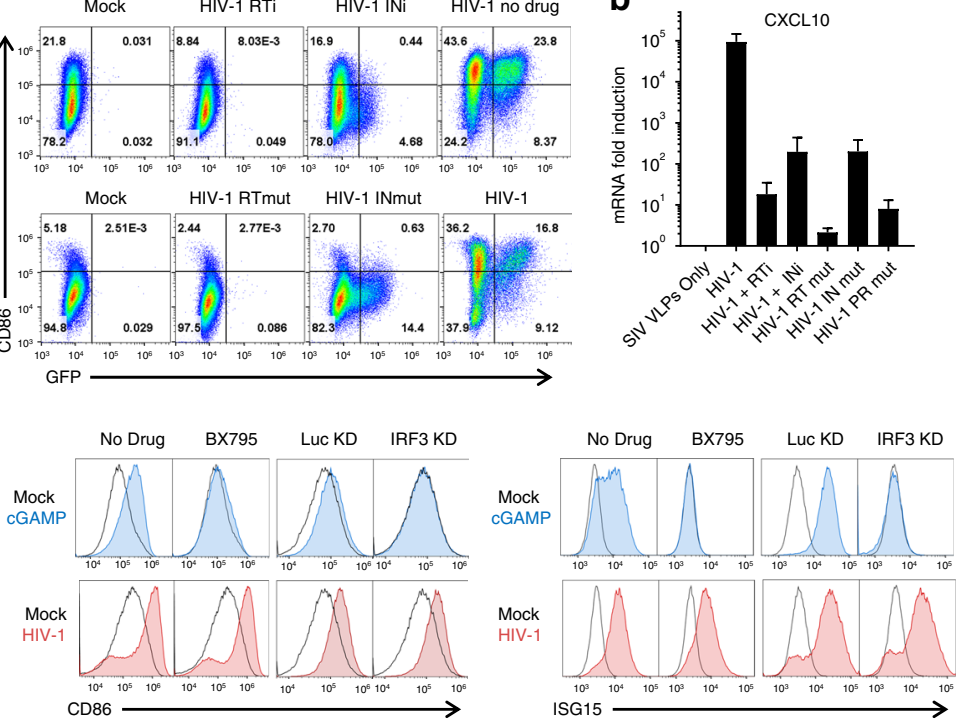

d
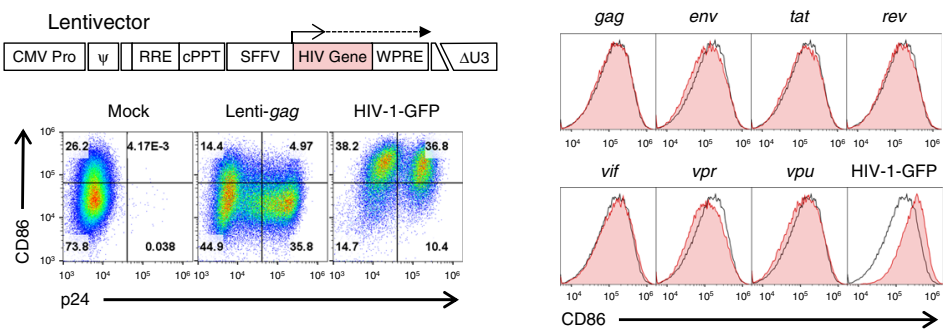

e
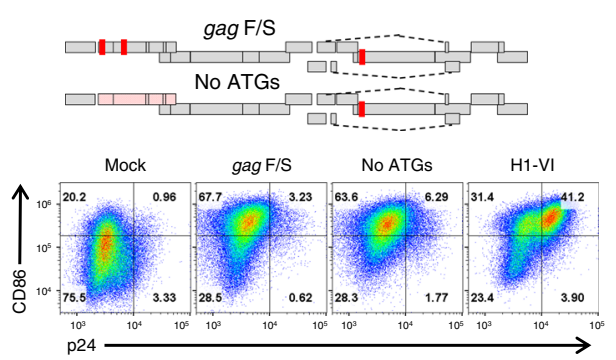

g
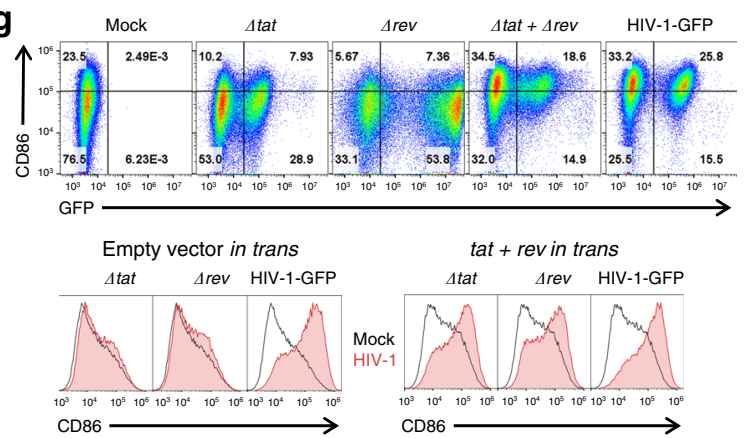

h Lentivector-U3+ f
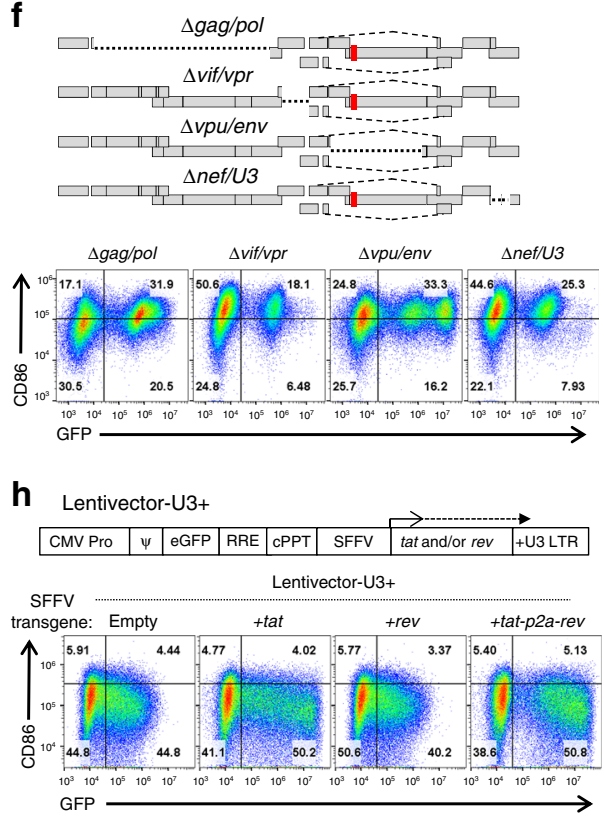

mechanism of innate immune activation was similar to that in DCs. As with DCs, HIV-1-GFP bearing the $\Delta g a g / p o l$ deletion activated macrophages and $\mathrm{CD}^{+}{ }^{+} \mathrm{T}$ cells (Fig. $4 \mathrm{~b}$ and Supplementary Fig. 4a, d). Also in agreement with the DC results, neither the minimal lentivector, nor HIV-1-GFP bearing mutations in integrase, tat, or rev, matured any of the three cell types (Fig. $4 \mathrm{~b}$ and Supplementary Fig. 4a, d).

$\mathrm{CD} 4^{+} \mathrm{T}$ cells were infected with either macrophage-tropic or $\mathrm{T}$ cell-tropic HIV-1 to determine whether replication-competent HIV-1 was similarly capable of innate immune activation in these cells, in the absence of VSV G. As with DCs, innate immune activation, as detected by MX1 and ISG15 upregulation, was observed in cells productively infected with HIV-1, but not with minimal lentivector (Fig. $4 \mathrm{c}$ and Supplementary Fig. 4g, h). Finally, to test the effect of HIV-1 proviral RNA on non-activated T cells, CD $4^{+} \mathrm{T}$ cells were co-transduced with Tet-HIV-1 and the rtTA3 vector, and cultured for 9 days in the absence of stimulation. Upon doxycycline treatment, T cells expressed GFP and MX1 (Fig. 4d and Supplementary Fig. 4i). As in DCs, dosedependent activation was observed with doxycycline (Fig. $4 \mathrm{~d}$ and 
Fig. 2 Native HIV-1 RNA regulation is necessary for DC maturation. a Assessment of GFP and CD86 by flow cytometry following transduction with, top, HIV-1-GFP in the presence of $5 \mu \mathrm{M}$ nevirapine (RTi), $10 \mu \mathrm{M}$ raltegravir (INi), or no drug, and, bottom, HIV-1-GFP bearing mutant RT-D185K/D186L (RTmut) or mutant IN-D116A (INmut). b qRT-PCR quantitation of CXCL10 mRNA from the same DCs as a. c DCs treated with $1 \mu$ M of the TBK1 inhibitor BX795, or expressing shRNAs targeting either IRF3 or luciferase control ${ }^{16}$, were challenged with $25 \mu \mathrm{g} / \mathrm{mL}$ cGAMP or HIV-1-GFP and assayed by flow cytometry for CD86 and ISG15. d Flow cytometry of DCs after transduction with minimal lentivectors expressing codon optimized HIV-1 genes; e, HIV-1-GFP in which translation was disrupted by two frameshifts in gag or by mutation of the first 14 AUGs in gag; $\mathbf{f}$, HIV-1-GFP bearing deletion mutations encompassing gag/pol, vif/vpr, vpu/env, or nef/U3-LTR; $\mathbf{g}$, HIV-1-GFP bearing mutations in tat or rev, co-transduced with both mutants, or co-transduced with minimal vector expressing tat and rev in trans; or $\mathbf{h}$ minimal lentivector with GFP in place of gag, SFFV promoter driving expression of tat, rev, or both, and repaired U3 in the 3' LTR. When an essential viral component was disrupted within HIV-1-GFP, the factor in question was provided in trans, either during assembly in transfected HEK293 cells, or within transduced DCs, as appropriate (see Methods). Shown are blood donor data representative of $n=6(\mathbf{a}, \mathbf{b}, \mathbf{e}, \mathbf{f}), n=12$ $(\mathbf{c}, \mathbf{g}, \mathbf{h}), n=8$ (d). To determine significance, the MFI of individual flow cytometry samples was calculated as fold-change versus control. When data from each donor replicate within a experiment were combined, the difference in MFI for all experimental vs control conditions was significant in all cases, $p<$ 0.01; one-way ANOVA, Dunnett's post-test against HIV-1-GFP for $\mathbf{a}, \mathbf{c}, \mathbf{d}, \mathbf{h}$ or lentivector control for $\mathbf{e}, \mathbf{f}$. qRT-PCR data are mean \pm SD ( $p<0.0001$; two-way ANOVA, Dunnett's post-test)

Supplementary Fig. 4i). These data indicate that innate immune activation by HIV-1, in all three cell types, requires integration, transcription, and Rev-dependent, HIV-1 intron-containing RNA.

\section{Discussion}

The HIV-1 LTR generates a single primary transcript that gives rise to over 100 alternatively spliced $\mathrm{RNAs}^{47}$. The full-length, unspliced, intron-bearing transcript acts as viral genomic RNA in the virion and as mRNA for essential gag- and pol-encoded proteins. Expression of the unspliced transcript requires specialized viral and cellular machinery, HIV-1 Rev and CRM1 ${ }^{45}$, in order to escape from processing by the spliceosome. Results here indicate that unspliced or partially spliced HIV-1 RNA is detected by human cells as a danger signal, as has been reported for inefficiently spliced mRNAs from transposable elements in distantly related eukaryotes ${ }^{48}$. Transposable elements are mutagenic to the host genome and it stands to reason that molecular features such as transcripts bearing multiple, inefficient splice signals characteristic of retrotransposons, would activate innate immune signaling pathways.

HIV-1 genomic RNA contains extensive secondary and higher order structures that could be detected by innate immune sensors. Our knockdown of IRF3 and inhibition of TBK1, both required for signal transduction of the RNA sensors RIG-I, MDA5, and TLR3, did not impede HIV-1 maturation of DCs (Fig. 2c and Supplementary Fig. 2b). Furthermore, we suppressed an extensive list of innate signaling pathways and sensors including knockdowns of IRF's 1, 5, 7, and 9, STAT's 1 and 2, as well as pharmacologic inhibition of CypA, PKR, c-Raf, IkBa, NF-kB, MEK1 +2, p38, JNK, Caspase 1, pan-Caspases, ASK1, eIF2a, IKKe, TAK1, or NLRP3 (Supplementary Fig. 2c and Supplementary Table 2). None of these perturbations had any effect on limiting innate immune activation by HIV-1, suggesting requirement for an alternative detection mechanism. Such mechanisms might include uncharacterized RNA sensors, more than one redundantly acting sensors, direct detection of stalled splicing machinery, or overload of the CRM1 nuclear export pathway itself.

The replication competent HIV-1 reservoir in memory CD4 ${ }^{+}$ $\mathrm{T}$ cells has a $44 \mathrm{wk}$ half-life and thus patients must take antiviral medication for life ${ }^{49}$. Long-lived, replication competent HIV-1 reservoirs in other cell types have not been clearly demonstrated, but these may also contribute to the HIV-1 reservoir ${ }^{3}$. The common genetic determinants in HIV-1 for maturation of CD4 ${ }^{+}$ $\mathrm{T}$ cells, macrophages, and DCs suggests that HIV-1 is detected by a mechanism that is conserved across cell types, and that this mechanism would be active in any cell type that possesses a transcriptionally active provirus. Data here show that proviruses need not be replication competent to contribute to inflammation.
Rather, HIV-1 transcription and export of unspliced RNA, regardless of replication competence, is sufficient to induce immune activation. This almost certainly contributes to systemic inflammation during acute or untreated chronic infection. Whether inflammation is activated in response to provirus expression in cells of patients on antiretroviral therapy remains to be determined. Consistent with our findings, $\mathrm{T}$ cell activation correlates directly with the level of cell-associated HIV-1 RNA in patients receiving antiretroviral therapy ${ }^{50}$. Finally, our data suggests that new drugs that block HIV-1 transcription, Tat-mediated transcriptional elongation, or Rev-mediated preservation of unspliced transcripts ${ }^{51}$, would limit inflammation, and offer an important addition to the current anti-HIV-1 drug armamentarium.

\section{Methods}

Data reporting. No statistical methods were used to predetermine sample size. The experiments were not randomized. The investigators were not blinded to allocation during experiments and outcome assessment.

Plasmids. The plasmids used here were either previously described or generated using standard cloning methods ${ }^{16}$. The full list of plasmids used here, along with their purpose and characteristics, is provided in Supplementary Table 1. All plasmid DNAs with complete nucleotide sequence files are available at www. addgene.com.

Cell culture. Cells were cultured at $37^{\circ} \mathrm{C}$ in $5 \% \mathrm{CO}_{2}$ humidified incubators and monitored for mycoplasma contamination using the Lonza Mycoplasma Detection kit by Lonza (LT07-318). HEK293 cells (ATCC) were used for viral production and were maintained in DMEM supplemented with 10\% FBS, $20 \mathrm{mM}$ L-glutamine (ThermoFisher), $25 \mathrm{mM}$ HEPES pH 7.2 (SigmaAldrich), $1 \mathrm{mM}$ sodium pyruvate (ThermoFisher), and $1 \times$ MEM non-essential amino acids (ThermoFisher). Cytokine conditioned media was produced from HEK293 cells stably transduced with pAIP-hGMCSF-co (Addgene \#74168), pAIP-hIL4-co (Addgene \#74169), or pAIPhIL2 (Addgene \#90513), as previously described ${ }^{16}$

Leukopaks were obtained from anonymous, healthy, blood bank donors (New York Biologics). As per NIH guidelines (http://grants.nih.gov/grants/policy/hs/ faqs_aps_definitions.htm), experiments with these cells were declared non-human subjects research by the UMMS IRB. PBMCs were isolated from leukopaks by gradient centrifugation on Histopaque-1077 (Sigma-Aldrich). CD14 ${ }^{+}$ mononuclear cells were isolated via positive selection using anti-CD14 antibody microbeads (Miltenyi). Enrichment for $\mathrm{CD}_{14}{ }^{+}$cells was routinely $>98 \%$.

To generate DCs or macrophages, $\mathrm{CD} 14^{+}$cells were plated at a density of 1 to $2 \times 10^{6}$ cells $/ \mathrm{mL}$ in RPMI-1640 supplemented with $5 \%$ heat inactivated human $\mathrm{AB}^{+}$serum (Omega Scientific, Tarzana, CA), $20 \mathrm{mM}$ L-glutamine, $25 \mathrm{mM}$ HEPES $\mathrm{pH} 7.2,1 \mathrm{mM}$ sodium pyruvate, and $1 \times$ MEM non-essential amino acids (RPMIHS complete) in the presence of cytokines that promote differentiation. DCs were generated by culturing monocytes for 6 days in the presence of 1:100 cytokineconditioned media containing human GM-CSF and human IL-4. DC preparations were consistently $>99 \%$ DC-SIGNhigh, CD11 $c^{\text {high }}$, and CD14low by flow cytometry. Macrophages were generated by culturing for 7 days with GM-CSF conditioned media in the absence of IL- 4 , and were routinely $>99 \%$ CD11b. CD4 ${ }^{+} \mathrm{T}$ cells were isolated from PBMCs that had been depleted of $\mathrm{CD} 14^{+}$cells, as above, using antiCD4 microbeads (Miltenyi), and were $>99 \% \mathrm{CD}^{+} . \mathrm{CD}^{+} \mathrm{T}$ cells were then cultured in RPMI-1640 supplemented with $10 \%$ heat inactivated FBS, $20 \mathrm{mM} \mathrm{L}$ - 
a

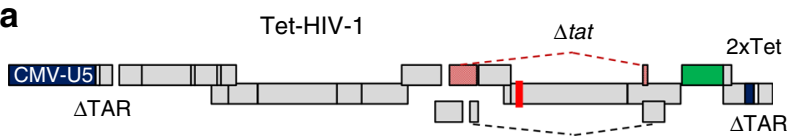

$500 \mathrm{ng} / \mathrm{mL}$ Dox

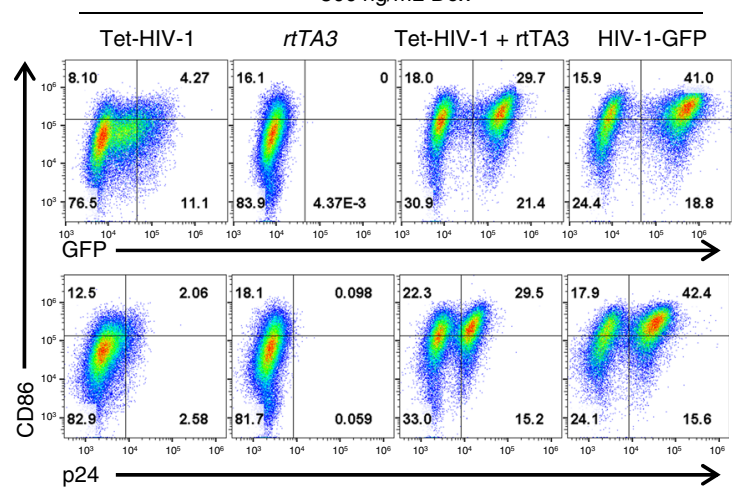

b

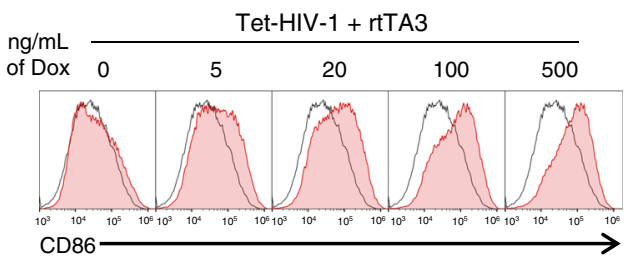

C
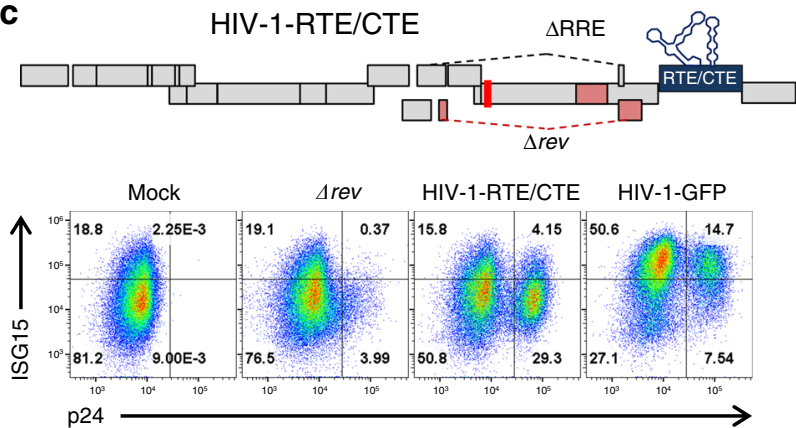

d

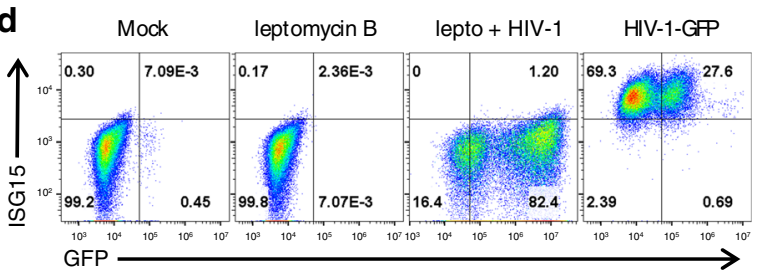

e

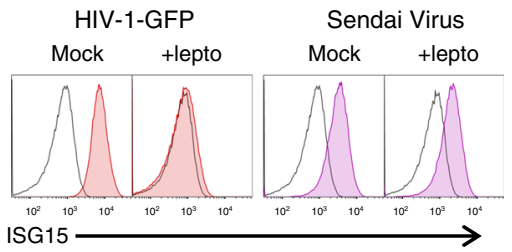

glutamine, $25 \mathrm{mM}$ HEPES $\mathrm{pH}$ 7.2, $1 \mathrm{mM}$ sodium pyruvate, $1 \times$ MEM non-essential amino acids (RPMI-FBS complete), and 1:2000 IL-2 conditioned media. Cells from particular donors were excluded from experiments if percent enrichment deviated more than $5 \%$ from the numbers mentioned above, or if there was no increase in activation markers in response to control stimuli (LPS, Sendai virus, and wild-type HIV-1-GFP).

HIV-1 vector production. HEK293E cells were seeded at $75 \%$ confluency in sixwell plates and transfected with $6.25 \mu \mathrm{L}$ Transit LT1 lipid reagent (Mirus) in $250 \mu \mathrm{L}$ Opti-MEM (Gibco) with $2.25 \mu \mathrm{g}$ total plasmid DNA. 2-part HIV-1 vectors based on HIV-1-GFP ${ }^{29}$ and described in detail in Supplementary Table 1 were
Fig. 3 Rev-mediated RNA export is necessary for DC maturation but Tat is dispensable. a Optimized $2 \times$ Tet operator $^{43}$ was cloned into the $3^{\prime} L T R$ of HIV-1-GFP $\Delta$ tat to generate Tet-HIV-1; the strand-transfer reactions that occur during reverse transcription generate a Tet-regulated 5 '-LTR in the provirus. DCs transduced with Tet-HIV-1, rtTA3, or both, were treated for $3 \mathrm{~d}$ with $500 \mathrm{ng} / \mathrm{mL}$ doxycycline and assayed by flow cytometry for p24, GFP, and CD86. b DCs co-transduced with Tet-HIV-1 and rtTA3 were treated with increasing concentrations of doxycycline. c To generate HIV-1RTE/CTE, the RTEm26CTE element ${ }^{44}$ was cloned in place of nef in HIV-1GFP $\Delta r e v / \triangle R R E$. DCs were transduced with the indicated vectors and assessed for P24 and ISG15 by flow cytometry. d DCs were treated with 25 $\mathrm{nM}$ leptomycin B, transduced with HIV-1-GFP, and assessed for GFP and ISG15 by flow cytometry. e DCs were treated with $25 \mathrm{nM}$ leptomycin B, transduced with HIV-1-GFP or infected with Sendai virus (SeV), and assessed for ISG15 by flow cytometry. Shown are blood donor data representative of $n=10(\mathbf{a}, \mathbf{c}), n=4(\mathbf{b}), n=6(\mathbf{d}, \mathbf{e})$. To determine significance, the MFI of individual flow cytometry samples was calculated as fold-change versus control. When data from each donor replicate within a experiment was combined, the difference in MFI for all experimental vs control conditions was significant in all cases, $p<0.01$; one-way ANOVA, Dunnett's post-test against dox negative control for $\mathbf{a}, \mathbf{b}$ or HIV-1-GFP for $\mathbf{c}-\mathbf{e}$

transfected at a 7:1 ratio in terms of $\mu$ gs of HIV-1 plasmid DNA to pMD2.G VSV $\mathrm{G}$ expression plasmid $\mathrm{DNA}^{16}$. Three-part lentivectors were produced by transfection of the lentivector genome, psPAX2 GagPol vector, and pMD2.G, at a DNA ratio of 4:3:1. These also include 2-part HIV-1-GFP constructs that are mutated in such a way as to prevent GagPol, Tat, or Rev production. As these would be defective for viral production, psPAX2 was included in the transfections at the same 4:3:1 ratio. VPX-bearing SIV-VLPs were produced by transfection at a 7:1 plasmid ratio of SIV3+ to pMD2.G ${ }^{16}$. Twelve hours after transfection, media was changed to the specific media for the cells that were to be transduced. Viral supernatant was harvested 2 days later, filtered through a $0.45 \mu \mathrm{m}$ filter, and stored at $4{ }^{\circ} \mathrm{C}$.

Virions in the transfection supernatant were quantified by a PCR-based assay for reverse transcriptase activity ${ }^{16}$. Five microliter transfection supernatant were

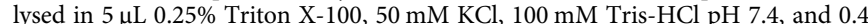
$\mathrm{U} / \mu \mathrm{L}$ RNase inhibitor (RiboLock, ThermoFisher). Viral lysate was then diluted 1:100 in a buffer of $5 \mathrm{mM}\left(\mathrm{NH}_{4}\right)_{2} \mathrm{SO}_{4}, 20 \mathrm{mM} \mathrm{KCl}$, and $20 \mathrm{mM}$ Tris- $\mathrm{HCl} \mathrm{pH} 8.3$. $10 \mu \mathrm{L}$ was then added to a single-step, RT PCR assay with $35 \mathrm{nM}$ MS2 RNA (IDT) as template, $500 \mathrm{nM}$ of each primer (5'-TCCTGCTCAACTTCCTGTCGAG-3' and $5^{\prime}$-CACAGGTCAAACCTCCTAGGAATG-3'), and hot-start Taq (Promega) in a buffer of $20 \mathrm{mM}$ Tris-Cl pH 8.3, $5 \mathrm{mM}\left(\mathrm{NH}_{4}\right)_{2} \mathrm{SO}_{4}, 20 \mathrm{mM} \mathrm{KCl}, 5 \mathrm{mM} \mathrm{MgCl}_{2}, 0.1$ $\mathrm{mg} / \mathrm{mL}$ BSA, 1/20,000 SYBR Green I (Invitrogen), and $200 \mu \mathrm{M}$ dNTPs. The RTPCR reaction was carried out in a Biorad CFX96 cycler with the following parameters: $42^{\circ} \mathrm{C} 20 \mathrm{~min}, 95^{\circ} \mathrm{C} 2 \mathrm{~min}$, and 40 cycles $\left[95^{\circ} \mathrm{C}\right.$ for $5 \mathrm{~s}, 60^{\circ} \mathrm{C} 5 \mathrm{~s}, 72^{\circ} \mathrm{C}$ for $15 \mathrm{~s}$ and acquisition at $80^{\circ} \mathrm{C}$ for $5 \mathrm{~s}$ ]. Two-part vectors typically yielded $10^{7} \mathrm{RT}$ units $/ \mu \mathrm{L}$, and 3 part vector transfections yielded $10^{6} \mathrm{RT}$ units $/ \mu \mathrm{L}$.

Transductions. $10^{6} \mathrm{DCs} / \mathrm{mL}$, or $5 \times 10^{5}$ macrophages $/ \mathrm{mL}$, were plated into RPMIHS complete with $\mathrm{Vpx}^{+}$SIV-VLP transfection supernatant added at a dilution of 1:6. After $2 \mathrm{~h}, 10^{8} \mathrm{RT}$ units of viral vector was added. In some cases, drugs were added to the culture media as specified in Supplementary Table 2. In most cases, transduced DC were harvested for analysis 3 days following challenge. For gene knockdown or for expression of factors in trans, $2 \times 10^{6} \mathrm{CD} 14^{+}$monocytes $/ \mathrm{mL}$ were transduced directly following magnetic bead isolation with 1:6 volume of SIVVLPs and 1:6 volume of vector. When drug selection was required, $4 \mu \mathrm{g} / \mathrm{mL}$ puromycin was added 3 days after monocyte transduction and cells were selected for 3 days. SIV-VLPs were re-administered in all cases with HIV-1 or lentivector challenge. For DCs in Tet-HIV-1 experiments, fresh monocytes were SIV-VLP treated and co-transduced with rtTA3 and Tet-HIV-1. DCs were harvested 6 days later and treated with indicated doxycycline concentrations.

For deoxynucleoside-assisted transductions, DCs were plated at $10^{6} \mathrm{DCs} / \mathrm{mL}$ and treated with $2 \mathrm{mM}$ of combined deoxynucleosides for $2 \mathrm{~h}$ before transduction with HIV-1. Deoxynucleosides were purchased from Sigma-Aldrich (2 deoxyguanosine monohydrate, cat\# D0901; thymidine, cat\# T1895; 2 deoxyadenosine monohydrate, cat\# D8668; 2' deoxycytidine hydrochloride, cat\# D0776). A $100 \mathrm{mM}$ stock solution was prepared by dissolving each of the four nucleotides at $100 \mathrm{mM}$ in RPMI 1640 by heating the medium at $80^{\circ} \mathrm{C}$ for $15 \mathrm{~min}$.

$\mathrm{CD}^{+} \mathrm{T}$ cells were stimulated in RPMI-FBS complete with 1:2000 hIL-2 conditioned media and $5 \mu \mathrm{g} / \mathrm{mL}$ PHA-P. After 3 days, T cells were replated at $10^{6}$ cells/mL in RPMI-FBS complete with hIL-2. Cells were transduced with $10^{8} \mathrm{RT}$ units of viral vector per $10^{6}$ cells and assayed 3 days later. T cells were cotransduced with rtTA3 and Tet-HIV-1 every day for 3 days after PHA stimulation. Cells were then replated in RPMI-FBS complete with hIL-2. Transduced T cells 
a

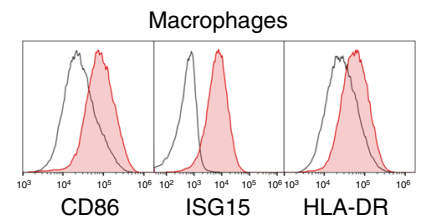

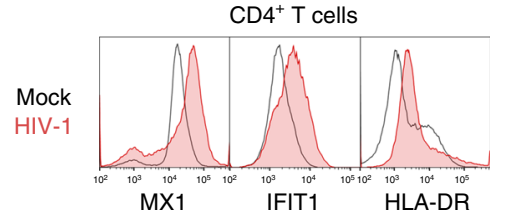

$\mathrm{MX1}$

IFIT1
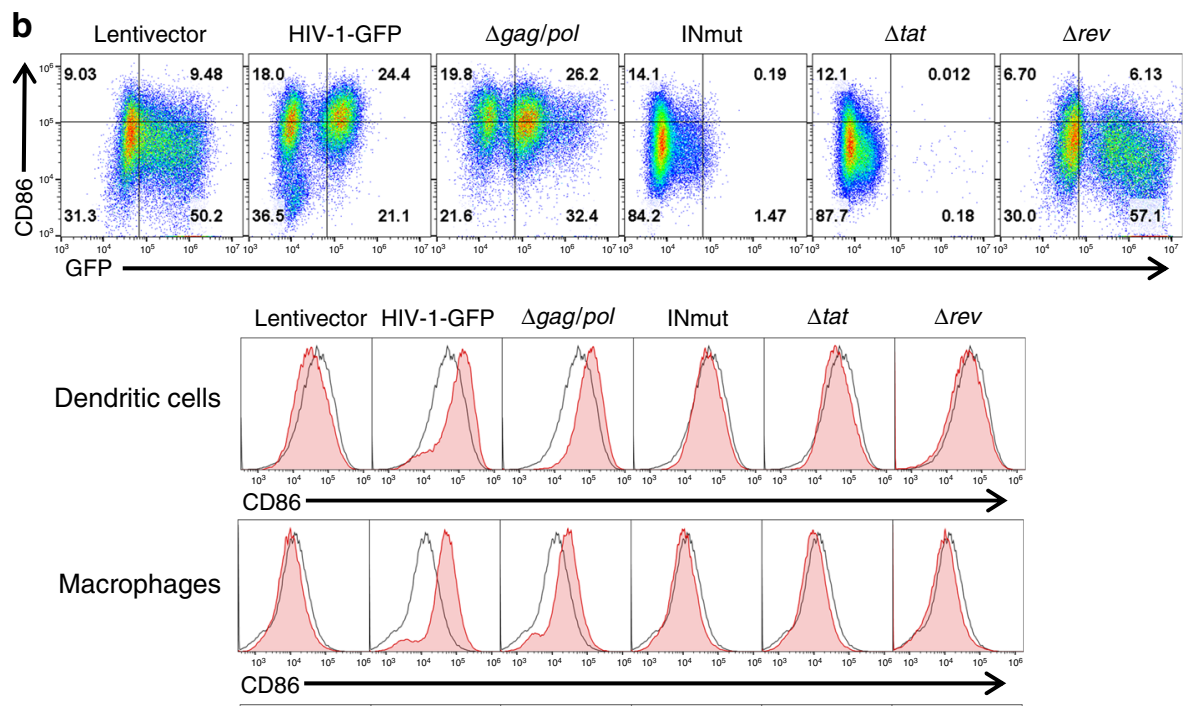

$\mathrm{CD}^{+} \mathrm{T}$ cells

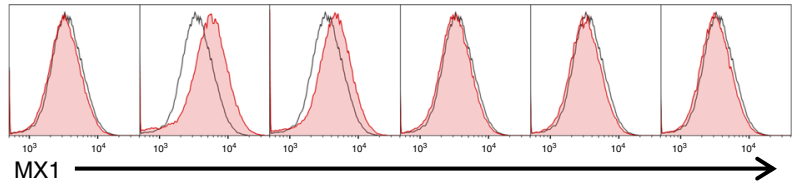

C

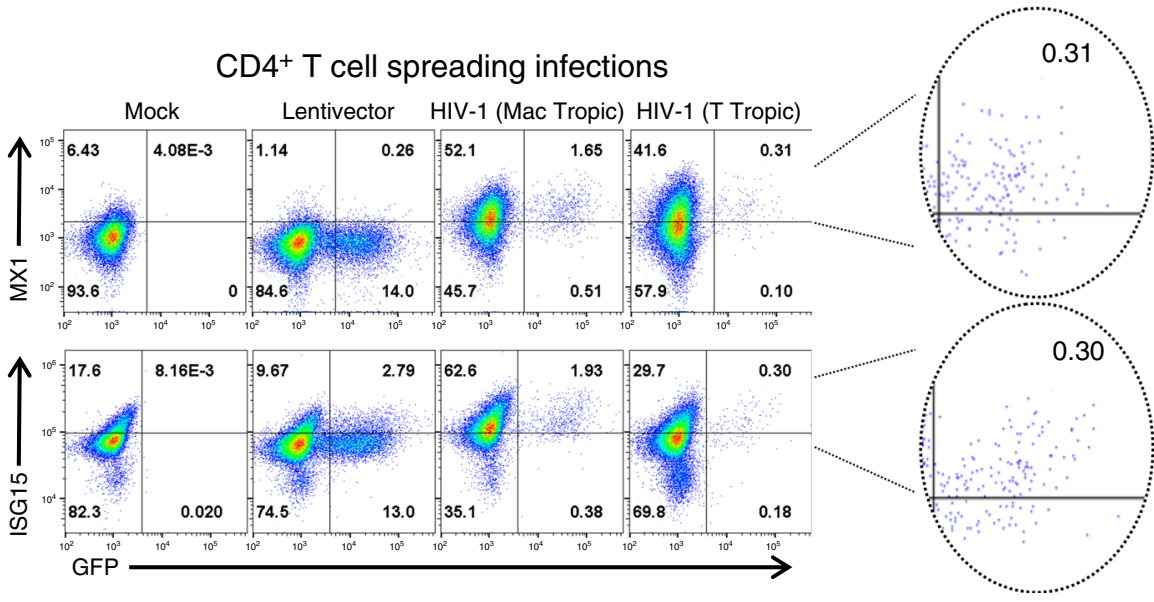

d

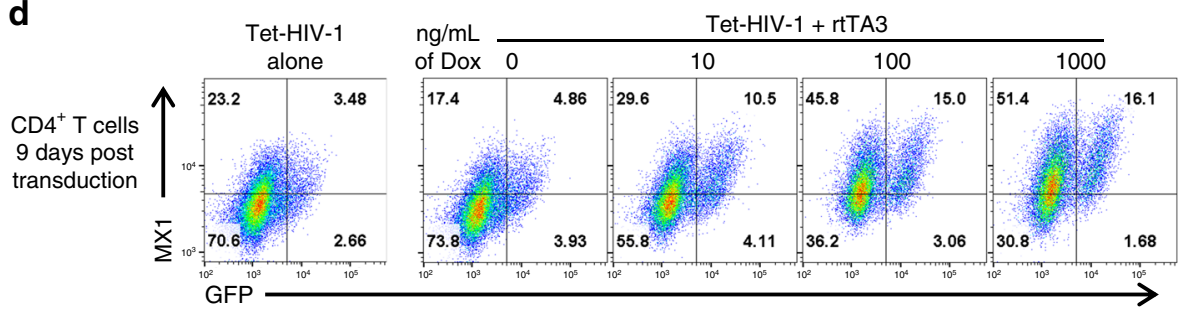

were cultured for 9 days with fresh media added at day 5. After 9 days, doxycycline was added at the indicated concentrations and assayed 3 days later.

Non-HIV-1 challenge viruses. Sendai Virus Cantell Strain was purchased from Charles River Laboratories. Infections were performed with $200 \mathrm{HA}$ units $/ \mathrm{mL}$ on DCs for 3 days before assay by flow cytometry.
Spreading infections. DCs were plated at $10^{6} \mathrm{DCs} / \mathrm{mL}$, in RPMI-HS complete media, with or without $\mathrm{Vpx}^{+}$SIV-VLP transfection supernatant added at a dilution of 1:6. After $2 \mathrm{~h}, 10^{8} \mathrm{RT}$ units of HEK-293 transfection supernatant of either NL43-GFP-JRFL or NL4-3-GFP-JRCSF was added. Each is a construct of pNL4-3 in which $e n v$ was replaced from the end of the signal peptide (therefore retaining the NL4-3 signal peptide and Vpu sequence) to the stop codon with either macrophage-tropic JR-FL env (GenBank: U63632.1) or T cell-tropic JR-CSF env 
Fig. 4 Innate immune activation in macrophages and CD4 ${ }^{+}$T cells. a Macrophages and CD4 ${ }^{+} \mathrm{T}$ cells were transduced with HIV-1-GFP and assayed 3 days later for the indicated activation markers. b DCs, macrophages, and CD4 ${ }^{+} \mathrm{T}$ cells were challenged with HIV-1-GFP or the indicated mutants. When an essential viral component was disrupted within HIV-1-GFP, the factor in question was provided in trans during assembly in transfected HEK293 cells, as appropriate (see Methods). The upper panel shows flow cytometry of the DCs for GFP and CD86. The histograms show CD86 for DCs and macrophages or $\mathrm{MX} 1$ for $\mathrm{CD} 4^{+} \mathrm{T}$ cells. c 12-day spreading infections on $\mathrm{CD} 4^{+} \mathrm{T}$ cells with either macrophage-tropic or $\mathrm{T}$ cell-tropic, replication-competent $\mathrm{HIV}-1$. d $\mathrm{CD} 4{ }^{+} \mathrm{T}$ cells were stimulated for 3 days with PHA and IL2, and transduced with Tet-HIV-1 and rtTA3. Cells were then cultured without stimulation for 9 days. Doxycycline was then added at the indicated concentrations. Cells were assayed for GFP and MX1 3 days later. Shown are blood donor data representative of $n=6(\mathbf{a}), n=4(\mathbf{b}-\mathbf{d})$. To determine significance, the MFI of individual flow cytometry samples was calculated as fold-change versus control. The exception being $\mathbf{c}$ where the MFI of only GFP + cells was compared. When data from each donor replicate within a experiment were combined, the difference in MFI for all experimental vs control conditions was significant in all cases, $p<0.01$; one-way ANOVA, Dunnett's post-test against lentivector control for a-c or dox control for $\mathbf{d}$

(GenBank: M38429.1). Every 3 days (for a total of 12 days) samples were harvested for detection of viral RT activity in supernatant and flow cytometry assessment.

$\mathrm{CD}^{+} \mathrm{T}$ cells were stimulated in RPMI-FBS complete with 1:2000 hIL-2 conditioned media and $5 \mu \mathrm{g} / \mathrm{mL}$ PHA-P. After 3 days, T cells were replated at $10^{6}$ cells $/ \mathrm{mL}$ in RPMI-FBS complete with hIL-2 and transduced with $10^{7} \mathrm{RT}$ units of NL4-3-GFP with JRFL (mac tropic) or JRCSF (T tropic) env. Cells were harvested every 3 days (for a total of 12 days) and assayed for infectivity and activation via flow cytometry.

Supernatant transfer experiments. Supernatant from HIV-1-GFP and minimal lentivector challenged DCs was harvested 3 days post transduction. This was centrifuged at $500 \times g$ for 5 mins and filtered through a $0.45 \mu \mathrm{m}$ filter to remove cell debris. DCs of the same individual donors were then treated with the harvested supernatant at a dilution of 1:1000 ( $1 \mu \mathrm{L}$ in $1 \mathrm{~mL}$ of culture). This was performed in the presence of nevirapine to inhibit secondary infection from remaining, cell free virions. Treatments of supernatants of HIV-1 challenged DCs were compared directly to unchallenged DC supernatant treatments.

Cytokine analysis. Supernatants from DCs were collected 3 days following transduction with HIV-1-GFP or minimal lentivector. Supernatant was spun at $500 \times g$ for 5 mins and filtered through a $0.45 \mu \mathrm{m}$ filter. Multiplex soluble protein analysis was carried out by Eve Technologies (Calgary, AB, Canada).

qRT-PCR. Total RNA was isolated from $5 \times 10^{5}$ DCs using RNeasy Plus Mini (Qiagen) with Turbo DNase (ThermoFisher) treatment between washes. Firststrand synthesis used Superscript III Vilo Master mix (Invitrogen) with random hexamers. qPCR was performed in $20 \mu \mathrm{L}$ using $1 \times$ TaqMan Gene Expression Master Mix (Applied Biosystems), $1 \mu \mathrm{L}$ cDNA, and $1 \mu \mathrm{L}$ TaqMan Gene Expression Assays (ThermoFisher) specified in Supplementary Table 3. Amplification was on a CFX96 Real Time Thermal Cycler (Bio-Rad) using the following program: $95^{\circ} \mathrm{C}$ for $10 \mathrm{~min}\left[45 \mathrm{cycles}\right.$ of $95^{\circ} \mathrm{C}$ for $15 \mathrm{~s}$ and $60^{\circ} \mathrm{C}$ for $60 \mathrm{~s}$. Housekeeping gene OAZ1 was used as control ${ }^{16}$.

Flow cytometry. $10^{5}$ cells were surface stained in FACS buffer (PBS, $2 \%$ FBS, $0.1 \%$ Sodium Azide), using the antibodies in Supplementary Table 4. All antibodies were used at a dilution of 1:100 with the exception of the p24 antibody (KC57 BeckmanCoulter) which was used at a 1:200 dilution. Cells were then fixed in a 1:4 dilution of BD Fixation Buffer and assayed on a BD C6 Accuri. BD Biosciences Fixation and Permeabilization buffers were utilized for intracellular staining. Data was analyzed in FlowJo.

Sampling. All individual experiments were performed with biological duplicates, using cells isolated from two different blood donors. Flow cytometry plots in the figures show representative data taken from experiments performed with cells from the number of donors indicated in the figure legends.

Statistical analysis. Experimental $n$ values and information regarding specific statistical tests can be found in the figure legends. The mean fluorescence intensity for all live cells analyzed under a given condition was calculated as fold-change to negative control/mock. The exception to this methodology was in Figs. $1 \mathrm{~g}$ and $4 \mathrm{c}$ where the percent infected cells was too low to use MFI for the bulk population; in these cases MFI was determined for the subset of cells within the GFP+ gate. Significance of flow cytometry data was determined via one-way ANOVA. A Dunnett's post-test for multiple comparisons was applied, where MFI fold change was compared to either mock treatment or positive treatment depending on the experimental question. QRT-PCR and luminex data was analyzed via two-way Anova, with Dunnett's post-test comparing all samples to mock. All ANOVAs were performed using PRISM 7.02 software (GraphPad Software, La Jolla, CA).

\section{Data availability}

The plasmids described in Supplementary Table 1, along with their complete nucleotide sequences, are available at www.addgene.com. The data that support the findings of this study are available from the corresponding author upon reasonable request. A reporting summary for this article is available as a Supplementary Information file.

Received: 1 May 2018 Accepted: 21 November 2018 Published online: 13 December 2018

\section{References}

1. Arts, E. J. \& Hazuda, D. J. HIV-1 antiretroviral drug therapy. Cold Spring Harb. Perspect. Med. 2, a007161 (2012).

2. Günthard, H. F. et al. Antiretroviral drugs for treatment and prevention of HIV infection in adults: 2016 recommendations of the International Antiviral Society-USA Panel. JAMA 316, 191-210 (2016).

3. Kandathil, A. J., Sugawara, S. \& Balagopal, A. Are T cells the only HIV-1 reservoir? Retrovirology 13, 86 (2016).

4. Siliciano, J. D. et al. Long-term follow-up studies confirm the stability of the latent reservoir for HIV-1 in resting CD4+T cells. Nat. Med. 9, 727-728 (2003).

5. Jiang, A.-P. et al. Human mucosal mast cells capture HIV-1 and mediate viral trans-infection of CD4+T cells. J. Virol. 90, 2928-2937 (2015).

6. Davey, R. T. Jr et al. HIV-1 and T cell dynamics after interruption of highly active antiretroviral therapy (HAART) in patients with a history of sustained viral suppression. Proc. Natl Acad. Sci. USA 96, 15109-15114 (1999).

7. Wang, D. et al. Evolution of drug-resistant viral populations during interruption of antiretroviral therapy. J. Virol. 85, 6403-6415 (2011).

8. Siliciano, J. D. \& Siliciano, R. F. Recent trends in HIV-1 drug resistance. Curr. Opin. Virol. 3, 487-494 (2013).

9. Johnson, V. A. et al. Update of the drug resistance mutations in HIV-1: March 2013. Top. Antivir. Med. 21, 6-14 (2013).

10. Freiberg, M. S. et al. HIV infection and the risk of acute myocardial infarction. JAMA Intern. Med. 173, 614-622 (2013).

11. Brenchley, J. M. et al. Microbial translocation is a cause of systemic immune activation in chronic HIV infection. Nat. Med. 12, 1365-1371 (2006).

12. Sinha, A. et al. Role of T-cell dysfunction, inflammation, and coagulation in microvascular disease in HIV. J. Am. Heart Assoc. 5, e004243 (2016).

13. Bruner, K. M. et al. Defective proviruses rapidly accumulate during acute HIV-1 infection. Nat. Med. 22, 1043-1049 (2016).

14. Wiegand, A. et al. Single-cell analysis of HIV-1 transcriptional activity reveals expression of proviruses in expanded clones during ART. Proc. Natl Acad. Sci. USA 114, E3659-E3668 (2017).

15. Luban, J. Innate immune sensing of HIV-1 by dendritic cells. Cell Host Microbe 12, 408-418 (2012).

16. Pertel, T. et al. TRIM5 is an innate immune sensor for the retrovirus capsid lattice. Nature 472, 361-365 (2011).

17. Lascano, J., Uchil, P. D., Mothes, W. \& Luban, J. TRIM5 retroviral restriction activity correlates with the ability to induce innate immune signaling. J. Virol. 90, 308-316 (2016).

18. Postler, T. S. \& Desrosiers, R. C. The cytoplasmic domain of the HIV-1 glycoprotein gp41 induces NF-KB activation through TGF- $\beta$-activated kinase 1. Cell. Host. Microbe 11, 181-193 (2012).

19. Fonteneau, J.-F. et al. Human immunodeficiency virus type 1 activates plasmacytoid dendritic cells and concomitantly induces the bystander maturation of myeloid dendritic cells. J. Virol. 78, 5223-5232 (2004).

20. O’Brien, M., Manches, O. \& Bhardwaj, N. Plasmacytoid dendritic cells in HIV infection. Adv. Exp. Med. Biol. 762, 71-107 (2013). 
21. Beignon, A.-S. et al. Endocytosis of HIV-1 activates plasmacytoid dendritic cells via Toll-like receptor-viral RNA interactions. J. Clin. Invest. 115, 3265-3275 (2005).

22. Solis, M. et al. RIG-I-mediated antiviral signaling is inhibited in HIV-1 infection by a protease-mediated sequestration of RIG-I. J. Virol. 85, 1224-1236 (2011).

23. Berg, R. K. et al. Genomic HIV RNA induces innate immune responses through RIG-I-dependent sensing of secondary-structured RNA. PLoS ONE 7, e29291 (2012).

24. Gao, D. et al. Cyclic GMP-AMP synthase is an innate immune sensor of HIV and other retroviruses. Science 341, 903-906 (2013).

25. Vermeire, J. et al. HIV Triggers a cGAS-Dependent, Vpu- and Vpr-Regulated Type I Interferon Response in CD4+T Cells. Cell Rep. 17, 413-424 (2016).

26. Lahaye, X. et al. The capsids of HIV-1 and HIV-2 determine immune detection of the viral cDNA by the innate sensor cGAS in dendritic cells. Immunity 39, 1132-1142 (2013).

27. Manel, N. et al. A cryptic sensor for HIV-1 activates antiviral innate immunity in dendritic cells. Nature 467, 214-217 (2010).

28. Gringhuis, S. I., et al HIV-1 blocks the signaling adaptor MAVS to evade antiviral host defense after sensing of abortive HIV-1 RNA by the host helicase DDX3. Nat. Immunol. 18, 225-235 (2017).

29. He, J. et al. CCR3 and CCR5 are co-receptors for HIV-1 infection of microglia. Nature 385, 645-649 (1997).

30. Landau, N. R. The innate immune response to HIV-1: to sense or not to sense. DNA Cell Biol. 33, 271-274 (2014).

31. Rasaiyaah, J. et al. HIV-1 evades innate immune recognition through specific cofactor recruitment. Nature 503, 402-405 (2013).

32. Goujon, C. et al. With a little help from a friend: increasing HIV transduction of monocyte-derived dendritic cells with virion-like particles of SIV(MAC). Gene Ther. 13, 991-994 (2006).

33. Reis e Sousa, C. Dendritic cells in a mature age. Nat. Rev. Immunol. 6, 476-483 (2006).

34. Parrish, N. F. et al. Phenotypic properties of transmitted founder HIV-1. Proc. Natl Acad. Sci. USA 110, 6626-6633 (2013).

35. Deymier, M. J. et al. Heterosexual transmission of subtype C HIV-1 selects consensus-like variants without increased replicative capacity or interferon- $\alpha$ resistance. PLoS Pathog. 11, e1005154 (2015).

36. Salazar-Gonzalez, J. F. et al. Genetic identity, biological phenotype, and evolutionary pathways of transmitted/founder viruses in acute and early HIV1 infection. J. Exp. Med. 206, 1273-1289 (2009).

37. Reinhard, C., Bottinelli, D., Kim, B. \& Luban, J. Vpx rescue of HIV-1 from the antiviral state in mature dendritic cells is independent of the intracellular deoxynucleotide concentration. Retrovirology 11, 12 (2014).

38. Granelli-Piperno, A., Delgado, E., Finkel, V., Paxton, W. \& Steinman, R. M. Immature dendritic cells selectively replicate macrophagetropic (M-Tropic) human immunodeficiency virus type 1 , while mature cells efficiently transmit both M- and T-tropic virus to T cells. J. Virol. 72, 2733-2737 (1998).

39. De Iaco, A. \& Luban, J. Inhibition of HIV-1 infection by TNPO3 depletion is determined by capsid and detectable after viral cDNA enters the nucleus. Retrovirology 8, 98 (2011).

40. Henderson, G. J. et al. Interplay between single resistance-associated mutations in the HIV-1 protease and viral infectivity, protease activity, and inhibitor sensitivity. Antimicrob. Agents Chemother. 56, 623-633 (2012).

41. Craigie, R. \& Bushman, F. D. HIV DNA integration. Cold Spring Harb. Perspect. Med. 2, a006890 (2012).

42. Zufferey, R. et al. Self-inactivating lentivirus vector for safe and efficient in vivo gene delivery. J. Virol. 72, 9873-9880 (1998)

43. Das, A. T. \& Berkhout, B. Conditionally replicating HIV and SIV variants. Virus Res. 216, 66-75 (2016).

44. Smulevitch, S. et al. RTE and CTE mRNA export elements synergistically increase expression of unstable, Rev-dependent HIV and SIV mRNAs. Retrovirology 3, 1-9 (2006).
45. Fornerod, M., Ohno, M., Yoshida, M. \& Mattaj, I. W. CRM1 is an export receptor for leucine-rich nuclear export signals. Cell 90, 1051-1060 (1997)

46. Chemnitz, J., Turza, N., Hauber, I., Steinkasserer, A. \& Hauber, J. The karyopherin CRM1 is required for dendritic cell maturation. Immunobiology 215, 370-379 (2010).

47. Ocwieja, K. E. et al. Dynamic regulation of HIV-1 mRNA populations analyzed by single-molecule enrichment and long-read sequencing. Nucleic Acids Res. 40, 10345-10355 (2012).

48. Dumesic, P. A. et al. Stalled spliceosomes are a signal for RNAi-mediated genome defense. Cell 152, 957-968 (2013)

49. Crooks, A. M. et al. Precise quantitation of the latent HIV-1 reservoir: implications for eradication strategies. J. Infect. Dis. 212, 1361-1365 (2015).

50. El-Diwany, R. et al. Intracellular HIV-1 RNA and CD4+T-cell activation in patients starting antiretrovirals. AIDS 31, 1405-1414 (2017).

51. Mousseau, G. et al. The Tat inhibitor didehydro-cortistatin A prevents HIV-1 reactivation from latency. mBio 6, e00465 (2015).

\section{Acknowledgements}

We thank the 290 anonymous blood donors who contributed leukocytes to this project, and Ben Berkhout, Abraham Brass, Barbara Felber, Beatrice Hahn, Eric Hunter, Massimo Pizzato, and Didier Trono for reagents. This work was supported by NIH Grants 5R01AI111809, 5DP1DA034990, and 1R01AI117839, to J.L.

\section{Author contributions}

S.M.M. and J.L. designed the experiments and S.M.M., K.K., A.D., A.N., L.Y., and W.E.D. conducted them. All authors analyzed the data and S.M.M. and J.L. wrote the manuscript.

\section{Additional information}

Supplementary Information accompanies this paper at https://doi.org/10.1038/s41467 018-07753-2.

Competing interests: The authors declare no competing interests.

Reprints and permission information is available online at http://npg.nature.com/ reprintsandpermissions/

Publisher's note: Springer Nature remains neutral with regard to jurisdictional claims in published maps and institutional affiliations.

Open Access This article is licensed under a Creative Commons Attribution 4.0 International License, which permits use, sharing, adaptation, distribution and reproduction in any medium or format, as long as you give appropriate credit to the original author(s) and the source, provide a link to the Creative Commons license, and indicate if changes were made. The images or other third party material in this article are included in the article's Creative Commons license, unless indicated otherwise in a credit line to the material. If material is not included in the article's Creative Commons license and your intended use is not permitted by statutory regulation or exceeds the permitted use, you will need to obtain permission directly from the copyright holder. To view a copy of this license, visit http://creativecommons.org/ licenses/by/4.0/.

(C) The Author(s) 2018 